\title{
Bochner-type property on spaces of generalized almost periodic functions ${ }^{1}$
}

\section{J.M. Sepulcre and T. Vidal}

Department of Mathematics, University of Alicante, 03080-Alicante, Spain.

ABSTRACT Our paper is focused on spaces of generalized almost periodic functions which, as in classical Fourier analysis, are associated with a Fourier series with real frequencies. In fact, based on a pertinent equivalence relation defined on the spaces of almost periodic functions in Bohr, Stepanov, Weyl and Besicovitch's sense, we refine the Bochner-type property by showing that the condition of almost periodicity of a function in any of these generalized spaces can be interpreted in the way that, with respect to the topology of each space, the closure of its set of translates coincides with its corresponding equivalence class.

AMS Subject Classification: 42A75, 42A16, 42B05, 46Axx, 42Axx.

Key Words: Almost periodic functions, Besicovitch almost periodic functions, Stepanov almost periodic functions, Weyl almost periodic functions, Bochner's theorem, Approximation by trigonometric polynomials, Exponential sums, Fourier series, Bohr's equivalence relation.

\section{Introduction}

The theory of almost periodic functions was mainly created during the 1920's by the Danish mathematician H. Bohr (1887-1951). The definition given by Bohr of an almost periodic function is based upon two properly generalized concepts: the periodicity to the so-called almost periodicity, and the periodic distribution of periods to the so-called relative density of almost periods. Specifically, let $f(t)$ be a real or complex function of an unrestricted real variable $t$, the notion above of almost periodicity involves the fact that $f(t)$ must be continuous, and for every $\varepsilon>0$ there corresponds a number $l=l(\varepsilon)>0$ such that each interval of length $l$ contains a number $\tau$ satisfying $|f(t+\tau)-f(t)|<\varepsilon$ for all $t$. We will denote as $A P(\mathbb{R}, \mathbb{C})$ the space of almost periodic functions in the sense of this definition (Bohr's condition).

The theory of almost periodic functions opened a way to study a wide class of trigonometric series of the general type and even exponential series (in this context, we can cite among others the papers [3, 4, 5, 6, 8, 11]). Furthermore, it has many important applications in problems of ordinary differential equations, dynamical systems, stability theory and partial differential equations (see for example recent developments in $[10,12,13]$ ). A very important result of this theory is the approximation theorem according to which the class of almost periodic functions $A P(\mathbb{R}, \mathbb{C})$ coincides with the class of limit functions of uniformly

\footnotetext{
${ }^{1}$ This is a preprint of an article published in Mediterranean Journal of Mathematics. The final authenticated version is available online at: https://doi.org/10.1007/s00009-020-01628-x
} 
convergent sequences of trigonometric polynomials of the type

$$
a_{1} e^{i \lambda_{1} t}+\ldots+a_{n} e^{i \lambda_{n} t}
$$

with arbitrary real exponents $\lambda_{j}$ and arbitrary complex coefficients $a_{j}$. Moreover, an equivalent definition for $A P(\mathbb{R}, \mathbb{C})$, called normality, was provided by $\mathrm{S}$. Bochner (in fact, sometimes it is called the Bochner-type definition) and it makes use of the generalization of the relative compactness, in the sense of uniform convergence, of translates of a continuous periodic function. Specifically, $A P(\mathbb{R}, \mathbb{C})$ consists of the continuous functions $f: \mathbb{R} \mapsto \mathbb{C}$ such that, given any sequence $\left\{h_{j}\right\}_{j \geq 1}$ of real numbers, there exists a subsequence $\left\{h_{j_{k}}\right\}_{k \geq 1} \subset\left\{h_{j}\right\}_{j \geq 1}$ satisfying that the sequence of functions $\left\{f\left(t+h_{j_{k}}\right)\right\}_{k \geq 1}$ is uniformly convergent.

In the course of time, Bohr's theory has been developed by a number of outstanding mathematicians. Indeed, several variants and extensions of Bohr's concept were introduced by A. S. Besicovitch, W. Stepanov and H. Weyl. We next provide their description, in the following three paragraphs, as a means for the reader to readily review their historical development and identify the relationship between them. It is already worth keeping in mind that any generalized almost periodic function $f(t)$ which is considered in this paper (the same as in [3]) has a Fourier series in the form of a general trigonometric series $\sum a_{n} e^{i \lambda_{n} t}$, where $\lambda_{n} \in \mathbb{R}$ are called Fourier exponents and $a_{k}$ are the coefficients of $f$ (see $[3$, p. 104]).

In this respect, the first generalizations of almost periodic functions in Bohr's sense were given by Stepanov [16], who succeeded in removing the continuity restrictions and characterize this new class in terms of mean values over integrals of fixed length. To do this, let $M(\mathbb{R}, \mathbb{C})$ be the more general space of locally integrable maps $f$ from $\mathbb{R}$ into $\mathbb{C}$ satisfying $\sup \left\{\int_{x}^{x+1}|f(t)| d t: x \in \mathbb{R}\right\}<\infty$. It is not difficult to prove that $|f|_{M}:=\sup \left\{\int_{x}^{x+1}|f(t)| d t: x \in \mathbb{R}\right\}$ defines a norm on $M(\mathbb{R}, \mathbb{C})$ and, in fact, $M(\mathbb{R}, \mathbb{C})$ is a Banach space endowed with this norm. In this way, the space $S(\mathbb{R}, \mathbb{C})$ of almost periodic functions in Stepanov sense is the closure in $M(\mathbb{R}, \mathbb{C})$ of the set of trigonometric polynomials of the form (1). With this definition, $S(\mathbb{R}, \mathbb{C})$ is a Banach space over the complex field $\mathbb{C}$ and it is verified that $A P(\mathbb{R}, \mathbb{C}) \subset S(\mathbb{R}, \mathbb{C})$. In general, the norm

$$
\|f\|_{S^{p}}:=\sup \left\{\left(\int_{x}^{x+1}|f(t)|^{p} d t\right)^{1 / p}: x \in \mathbb{R}\right\}
$$

leads us to the spaces $S^{p}(\mathbb{R}, \mathbb{C}), 1 \leq p<\infty$. However, it is accomplished that $S^{p}(\mathbb{R}, \mathbb{C}) \subset S(\mathbb{R}, \mathbb{C})$, which tells us that the space $S(\mathbb{R}, \mathbb{C})$ is the richer space of almost periodic functions in Stepanov's sense. Furthermore, the spaces $S^{p}(\mathbb{R}, \mathbb{C})$ can also be characterized through a Bohr-type definition in the sense that a locally integrable map $f$ from $\mathbb{R}$ into $\mathbb{C}$ is in $S^{p}(\mathbb{R}, \mathbb{C})$ if and only if for every $\varepsilon>0$ there corresponds a relatively dense set of real numbers $\{\tau\}$ satisfying $\|f(t+\tau)-f(t)\|_{S^{p}}<\varepsilon$ (see [3, pp. 79,88]). In the same way, this notion is also equivalent in this case to the $S^{p}$-normality, i.e. the relative compactness, with respect to the topology given by $\|\cdot\|_{S^{p}}$, of the family of the translates $\{f(t+h)\}, h \in \mathbb{R}$ (see for example [2, Theorem 3.5]). 
A generalization of these functions was given by $\mathrm{H}$. Weyl through the spaces which we will denote as $W^{p}(\mathbb{R}, \mathbb{C}) \supset S^{p}(\mathbb{R}, \mathbb{C}), 1 \leq p<\infty$. Specifically, the functions in $W^{p}(\mathbb{R}, \mathbb{C})$ are obtained by the completion of the trigonometric polynomials of the form (1) with respect to the seminorm

$$
\|f\|_{W^{p}}:=\lim _{l \rightarrow \infty} \sup \left\{\left(\frac{1}{l} \int_{x}^{x+l}|f(t)|^{p} d t\right)^{1 / p}: x \in \mathbb{R}\right\} .
$$

Equivalently, a locally integrable map $f$ from $\mathbb{R}$ into $\mathbb{C}$ is in $W^{p}(\mathbb{R}, \mathbb{C})$ if and only if for every $\varepsilon>0$ there corresponds a relatively dense set $\{\tau\}$ of real numbers and a number $L_{0}>0$ satisfying

$$
\sup \left\{\left(\frac{1}{l} \int_{x}^{x+l}|f(t+\tau)-f(t)|^{p} d t\right)^{1 / p}: x \in \mathbb{R}\right\}<\varepsilon \forall \tau \in\{\tau\} \forall l \geq L_{0},
$$

i.e.

$$
\|f(t+\tau)-f(t)\|_{S_{l}^{p}}<\varepsilon \forall \tau \in\{\tau\} \forall l \geq L_{0},
$$

where $\|f\|_{S_{l}^{p}}:=\sup \left\{\left(\frac{1}{l} \int_{x}^{x+l}|f(t)|^{p} d t\right)^{1 / p}: x \in \mathbb{R}\right\}, l>0$. (See for example [3, pp. 82,88], [9, p. 5] or [2, Definition 4.1 and p. 140] where the functions in this space are also called equi-almost-periodic in the sense of Weyl). In this case, the analogous theorem for the $W^{p}$-normality is stated in the sense that a locally integrable map $f$ from $\mathbb{R}$ into $\mathbb{C}$ is in $W^{p}(\mathbb{R}, \mathbb{C})$ if and only if the family of the translates $\{f(t+h)\}, h \in \mathbb{R}$, is relatively compact with respect to the topology given by $\|\cdot\|_{S_{l}^{p}}$, for $l$ sufficiently large (see [2, Definition 4.2 and Theorem 4.12]). Furthermore, every function in the space $W^{p}(\mathbb{R}, \mathbb{C})$ satisfies the fact that the family of the translates $\{f(t+h)\}, h \in \mathbb{R}$, is relatively compact with respect to the topology given by $\|\cdot\|_{W^{p}}$ (see $[2$, Definition 4.5 and Theorem $4.25]$ ). However, $W^{p}(\mathbb{R}, \mathbb{C}$ ) is not a complete space (see for example $[7$, Section $2.3])$.

On the other hand, the Besicovitch spaces $B^{p}(\mathbb{R}, \mathbb{C}), 1 \leq p<\infty$, are obtained by the completion of the trigonometric polynomials of the form (1) with respect to the topology, which is weaker than that of the uniform convergence, given by the seminorms

$$
\|f\|_{B^{p}}:=\left(\limsup _{l \rightarrow \infty} \frac{1}{2 l} \int_{-l}^{l}|f(t)|^{p} d t\right)^{1 / p} .
$$

Equivalently, the functions in $B^{p}(\mathbb{R}, \mathbb{C})$ satisfy that for every $\varepsilon>0$ there corresponds a satisfactorily uniform set $\left\{\tau_{k}\right\}_{k \in \mathbb{Z}}$ of real numbers (i.e. there exists $l>0$ such that the ratio of the maximum number of terms of $\left\{\tau_{k}\right\}$ included in an interval of length $l$ to the minimum number is less than 2), with $\tau_{i}<\tau_{j}$ if 
$j<i$, such that $\left\|f\left(t+\tau_{k}\right)-f(t)\right\|_{B^{p}}<\varepsilon$ for each $k$, and that for every $c>0$

$\limsup _{T \rightarrow \infty}\left(\frac{1}{2 T} \int_{-T}^{T}\left(\limsup _{n \rightarrow \infty} \frac{1}{2 n+1} \sum_{k=-n}^{n} \frac{1}{c} \int_{x}^{x+c}\left|f\left(t+\tau_{k}\right)-f(t)\right|^{p} d t\right) d x\right)^{\frac{1}{p}}<\varepsilon$.

(See $[3$, pp. $78,95,100]$ or $\left[2\right.$, Theorem 5.12]). For this case, if $f \in B^{p}(\mathbb{R}, \mathbb{C})$ then it is accomplished the relative compactness, with respect to the topology given by $\|\cdot\|_{B^{p}}$, of the family of its translates $\{f(t+h)\}, h \in \mathbb{R}$ (see $[2$, Theorem $5.34])$.

It is worth noting that, in general, when we write that a function $f$ is in one of these spaces we do not have in mind the function $f$ itself, it does represent a whole class of equivalent functions according to the relation $f_{1} \simeq f_{2}$ given by the corresponding seminorm (which certainly becomes a norm on the spaces composed of equivalence classes). Moreover, the space $B^{1}(\mathbb{R}, \mathbb{C})$ is denoted by $B(\mathbb{R}, \mathbb{C})$ and it contains $A P(\mathbb{R}, \mathbb{C}), S(\mathbb{R}, \mathbb{C}), W(\mathbb{R}, \mathbb{C})$ and all spaces of almost periodic functions we have discussed so far. In fact, it is satisfied that $A P(\mathbb{R}, \mathbb{C}) \subset S^{2}(\mathbb{R}, \mathbb{C}) \subset W^{2}(\mathbb{R}, \mathbb{C}) \subset B^{2}(\mathbb{R}, \mathbb{C}) \subset B(\mathbb{R}, \mathbb{C})$ and $A P(\mathbb{R}, \mathbb{C}) \subset S(\mathbb{R}, \mathbb{C}) \subset W(\mathbb{R}, \mathbb{C}) \subset B(\mathbb{R}, \mathbb{C})$. Furthermore, for every function $f \in B(\mathbb{R}, \mathbb{C})$ there exists the mean value

$$
M(f)=\lim _{l \rightarrow \infty} \frac{1}{2 l} \int_{-l}^{l} f(t) d t
$$

and, at most, a countable set of values of $\lambda_{k} \in \mathbb{R}$ such that $a_{k}=a\left(f, \lambda_{k}\right)=$ $M\left(f(t) e^{-i \lambda_{k} t}\right) \neq 0$. In this way, the series $\sum_{k \geq 1} a_{k} e^{i \lambda_{k} t}$ is called the Fourier series of $f[8$, Section 4.2].

For our purposes, it will be worth taking into consideration that if an almost periodic function $f$ has as Fourier series $\sum_{k>1} a_{k} e^{i \lambda_{k} t}$, then $f$ can be approximated, with respect to its corresponding topology, by (Bochner-Fejér's) polynomials of the form $P_{k}^{f}(t)=\sum_{j \geq 1} p_{j, k} a_{j} e^{i \lambda_{j} t}$, where the terms $p_{j, k}$ only depend on the frequency $\lambda_{j}$ not on the function $f$ (see the Bochner-Fejér's method of summation [3, Chapter 1, Section 9], [3, p. 105, Theorem II] or [7, pp. 46-47]).

Besicovitch's generalization is interesting because, for this extension, the analogue of the Riesz-Fischer theorem is also valid, that is to say, any trigonometric series $\sum_{n \geq 1} a_{n} e^{i \lambda_{n} t}$, with $\sum_{n \geq 1}\left|a_{n}\right|^{2}<\infty$, is the Fourier series of a $B^{2}(\mathbb{R}, \mathbb{C})$ almost periodic function $\left[3\right.$, p. 110] (in this sense, $B^{2}(\mathbb{R}, \mathbb{C}$ ) is also called $A P_{2}(\mathbb{R}, \mathbb{C})$ in $\left.[8]\right)$. This is not the case for some Stepanov or Weyl functions [11]. Note that Riesz-Fischer theorem was used in [15] in order to obtain our results for these spaces (see the proof of [15, Lemma 1]).

Precisely, in the context of the Besicovitch almost periodic functions $B(\mathbb{R}, \mathbb{C})$, among which it was considered an equivalence relation in terms similar to Bohr's equivalence relation on general Dirichlet series (see [15, Definition 5]), the main result of [15] states that, given an almost periodic function in $B^{2}(\mathbb{R}, \mathbb{C})$, the limit points of the set of its translates are precisely the functions which are equivalent to it. Likewise, in terms of this equivalence relation on the space $A P(\mathbb{R}, \mathbb{C})$, the paper [14] refined Bochner's result in the sense that we proved 
that the condition of almost periodicity (in $A P(\mathbb{R}, \mathbb{C})$ ) is equivalent to that every sequence of translates has a subsequence that converges uniformly to an equivalent function.

In this paper we show that the Bochner-type property, which is satisfied for all these classes of generalized almost periodic functions, can be refined in the sense that the condition of almost periodicity (in $A P(\mathbb{R}, \mathbb{C}), S^{p}(\mathbb{R}, \mathbb{C}), W^{p}(\mathbb{R}, \mathbb{C})$ or $B^{p}(\mathbb{R}, \mathbb{C})$ ) implies that every sequence of translates has a subsequence that converges, with respect to the topology of the corresponding space, to an equivalent function (see Theorem 14 and Corollary 16). This means that, while it is true that the proofs of the intermediate results of this paper are notably different from those of $[14,15]$, the main results of both papers $[14,15]$ are now extended to all these spaces. In fact, we can go further by extending these results to every existing space of generalized almost periodic functions satisfying the appropriate conditions (see Remark 17).

\section{Preliminaries}

We shall refer to the expressions of the type

$$
P_{1}(p) e^{\lambda_{1} p}+\ldots+P_{j}(p) e^{\lambda_{j} p}+\ldots
$$

as exponential sums, where the frequencies $\lambda_{j}$ are complex numbers and the $P_{j}(p)$ are polynomials in $p$. In this paper we are going to consider some functions which are associated with a concrete subclass of these exponential sums, where the parameter $p$ will be changed by $t$ in the real case. In this way, as in [14], we take the following definition.

Definition 1 Let $\Lambda=\left\{\lambda_{1}, \lambda_{2}, \ldots, \lambda_{j}, \ldots\right\}$ be an arbitrary countable set of distinct real numbers, which we will call a set of exponents or frequencies. We will say that an exponential sum is in the class $\mathcal{S}_{\Lambda}$ if it is a formal series of type

$$
\sum_{j \geq 1} a_{j} e^{\lambda_{j} p}, a_{j} \in \mathbb{C}, \lambda_{j} \in \Lambda .
$$

We next consider the same equivalence relation on the classes $\mathcal{S}_{\Lambda}$ as that of [15, Definition 2].

Definition 2 Given an arbitrary countable set $\Lambda=\left\{\lambda_{1}, \lambda_{2}, \ldots, \lambda_{j}, \ldots\right\}$ of distinct real numbers, consider $A_{1}(p)$ and $A_{2}(p)$ two exponential sums in the class $\mathcal{S}_{\Lambda}$, say $A_{1}(p)=\sum_{j \geq 1} a_{j} e^{\lambda_{j} p}$ and $A_{2}(p)=\sum_{j \geq 1} b_{j} e^{\lambda_{j} p}$. We will say that $A_{1}$ is ${ }^{*}$-equivalent to $A_{2}$ (in that case, we will write $A_{1} \stackrel{*}{\sim} A_{2}$ ) if for each integer value $n \geq 1$, with $n \leq \sharp \Lambda$, there exists a $\mathbb{Q}$-linear map $\psi_{n}: V_{n} \rightarrow \mathbb{R}$, where $V_{n}$ is the $\mathbb{Q}$-vector space generated by $\left\{\lambda_{1}, \lambda_{2}, \ldots, \lambda_{n}\right\}$, such that

$$
b_{j}=a_{j} e^{i \psi_{n}\left(\lambda_{j}\right)}, j=1, \ldots, n .
$$


Let $G_{\Lambda}=\left\{g_{1}, g_{2}, \ldots, g_{k}, \ldots\right\}$ be a basis of the vector space over the rationals generated by a set $\Lambda$ of exponents, which implies that $G_{\Lambda}$ is linearly independent over the rationals and each $\lambda_{j}$ is expressible as a finite linear combination of terms of $G_{\Lambda}$, say

$$
\lambda_{j}=\sum_{k=1}^{q_{j}} r_{j, k} g_{k}, \text { for some } r_{j, k} \in \mathbb{Q} .
$$

By abuse of notation, we will say that $G_{\Lambda}$ is a basis for $\Lambda$. Moreover, we will say that $G_{\Lambda}$ is an integral basis for $\Lambda$ when $r_{j, k} \in \mathbb{Z}$ for any $j, k$. Now, the equivalence relation introduced in Definition 2 can be characterized in terms of a basis for $\Lambda$ (see the proof in [15, Proposition 1]).

Proposition 3 Given $\Lambda=\left\{\lambda_{1}, \lambda_{2}, \ldots, \lambda_{j}, \ldots\right\}$ a set of exponents, consider $A_{1}(p)$ and $A_{2}(p)$ two exponential sums in the class $\mathcal{S}_{\Lambda}$, say $A_{1}(p)=\sum_{j \geq 1} a_{j} e^{\lambda_{j} p}$ and $A_{2}(p)=\sum_{j \geq 1} b_{j} e^{\lambda_{j} p}$. Fixed a basis $G_{\Lambda}$ for $\Lambda$, for each $j \geq 1$ let $\mathbf{r}_{j}$ be the vector of rational components satisfying (3). Then $A_{1} \stackrel{*}{\sim} A_{2}$ if and only if for each integer value $n \geq 1$, with $n \leq \sharp \Lambda$, there exists a vector $\mathbf{x}_{n}=$ $\left(x_{n, 1}, x_{n, 2}, \ldots, x_{n, k}, \ldots\right) \in \mathbb{R}^{\sharp G_{\Lambda}}$ such that $b_{j}=a_{j} e^{<\mathbf{r}_{j}, \mathbf{x}_{n}>i}$ for $j=1,2, \ldots, n$.

Furthermore, if $G_{\Lambda}$ is an integral basis for $\Lambda$ then $A_{1} \stackrel{*}{\sim} A_{2}$ if and only if there exists $\mathbf{x}_{0}=\left(x_{0,1}, x_{0,2}, \ldots, x_{0, k}, \ldots\right) \in \mathbb{R}^{\sharp G_{\Lambda}}$ such that $b_{j}=a_{j} e^{<\mathbf{r}_{j}, \mathbf{x}_{0}>i}$ for every $j \geq 1$.

In particular, the coefficients of *-equivalent exponential sums have the same modulus [15, Remark 1].

In the last part of this section, we focus our attention on the following classes of finite exponential sums or trigonometric polynomials.

Definition 4 Let $\Lambda=\left\{\lambda_{1}, \ldots, \lambda_{n}\right\}$ be a set of $n \geq 1$ distinct real numbers. We will say that a function $f: \mathbb{R} \mapsto \mathbb{C}$ is in the class $\mathcal{P}_{\mathbb{R}, \Lambda}$ if it is of the form

$$
f(t)=a_{1} e^{i \lambda_{1} t}+\ldots+a_{n} e^{i \lambda_{n} t}, a_{j} \in \mathbb{C}, \lambda_{j} \in \Lambda, j=1, \ldots, n .
$$

It is clear that Definition 2 can be particularized to the classes $\mathcal{P}_{\mathbb{R}, \Lambda}$. Note that if the set of exponents $\Lambda$ is finite it is always feasible to find an integral basis for $\Lambda$.

In the context of trigonometric polynomials, we next prove the following result which will be very useful to get the main results in this paper.

Proposition 5 Given $\Lambda=\left\{\lambda_{1}, \lambda_{2}, \ldots, \lambda_{n}\right\}$ a finite set of exponents, let $f_{1}(t)=$ $\sum_{j=1}^{n} a_{j} e^{i \lambda_{j} t}$ and $f_{2}(t)=\sum_{j=1}^{n} b_{j} e^{i \lambda_{j} t}$ be two ${ }^{*}$-equivalent functions in the class $\mathcal{P}_{\mathbb{R}, \Lambda}$. Given $\varepsilon>0$, there exists a relatively dense set $A$ of real numbers such that

$$
\left\|f_{1}(t+\tau)-f_{2}(t)\right\|_{G} \leq \varepsilon \forall \tau \in A,
$$

where $G$ could be $S^{p}, S_{l}^{p}$, $W^{p}$ or $B^{p}$, with $1 \leq p<\infty$ and $l>0$. 
Proof. Fixed $\varepsilon>0$, by [15, Corollary 1] we assure the existence of a relatively dense set $A$ of real numbers $\tau$ such that $\left|f_{1}(t+\tau)-f_{2}(t)\right|<\varepsilon \forall t \in \mathbb{R}$. Consequently, by virtue of [3, p. 73] (or [2, p. 170]), every $\tau \in A$ satisfies

$$
\left\|f_{1}(t+\tau)-f_{2}(t)\right\|_{G} \leq \sup \left\{\left|f_{1}(t+\tau)-f_{2}(t)\right|: t \in \mathbb{R}\right\} \leq \varepsilon
$$

which proves the result.

We recall that if $\Lambda$ is a finite set of exponents and $\mathcal{G}$ is an equivalence class in $\mathcal{P}_{\mathbb{R}, \Lambda} /{ }^{*}$, then $\mathcal{G}$ is compact with respect to the topology of the uniform convergence (see [15, Proposition 3]).

\section{Generalized almost periodic functions in terms of an equivalence relation}

As we showed in the introduction, every space of generalized almost periodic functions is associated with a concrete distance or metric. When we wish refer to a function of one of these spaces or a distance without specifying a definite kind we shall write a $G$-a.p. function or $\|\cdot\|_{G}$, respectively, where $G$ could be $S^{p}, W^{p}, B^{p}$, with $1 \leq p<\infty$, or even $A P$ (associated with the uniform convergence).

Note that, by [3, p. 109], if two $G$-a.p. functions $f_{1}(t)$ and $f_{2}(t)$ have the same Fourier series, then $\left\|f_{1}(t)-f_{2}(t)\right\|_{G}=0$. Therefore, when we write that a function $f(t)$ is in these spaces we do not have in mind the function $f(t)$ itself, it does represent a whole class of equivalent functions. We will denote as $G(\mathbb{R}, \mathbb{C})$ this space of equivalence classes of $G$-a.p. functions.

For our purposes, it is also worth taking into consideration that the BochnerFejér's method of summation, which is very important in the class $A P(\mathbb{R}, \mathbb{C})$ (see, in this regard, [3, Chapter 1, Section 9]), can be transferred to the $G$ a.p. functions in the sense that if a function $f(t)$ belongs to $G(\mathbb{R}, \mathbb{C})$, then any Bochner-Fejér's sequence $P_{k}^{f}(t)$ satisfies $\left\|f(t)-P_{k}^{f}(t)\right\|_{G} \rightarrow 0$ as $k \rightarrow \infty$ (see [3, p. 105, Theorem II] or [7, pp. 46-47]).

We next focus our attention on the following classes of almost periodic functions in $G(\mathbb{R}, \mathbb{C})$.

Definition 6 Let $\Lambda=\left\{\lambda_{1}, \lambda_{2}, \ldots, \lambda_{j}, \ldots\right\}$ be an arbitrary countable set of distinct real numbers. We will say that a function $f: \mathbb{R} \rightarrow \mathbb{C}$ is in the class $\mathcal{F}_{G, \Lambda}$ if it is an almost periodic function in $G(\mathbb{R}, \mathbb{C})$ whose associated Fourier series is of the form

$$
\sum_{j \geq 1} a_{j} e^{i \lambda_{j} t}, a_{j} \in \mathbb{C}, \lambda_{j} \in \Lambda
$$

In terms of Definition 2, we can define an equivalence relation on the functions in the classes $\mathcal{F}_{G, \Lambda}$. More specifically, we establish the following definition. 
Definition 7 Given $\Lambda=\left\{\lambda_{1}, \lambda_{2}, \ldots, \lambda_{j}, \ldots\right\}$ a set of exponents, consider the functions $f_{1}, f_{2} \in G(\mathbb{R}, \mathbb{C})$ whose associated Fourier series are respectively given by

$$
\sum_{j \geq 1} a_{j} e^{i \lambda_{j} t} \text { and } \sum_{j \geq 1} b_{j} e^{i \lambda_{j} t}, a_{j}, b_{j} \in \mathbb{C}, \lambda_{j} \in \Lambda .
$$

We will say that $f_{1}$ is ${ }^{*}$-equivalent to $f_{2}$ if for each integer value $n \geq 1$, with $n \leq \sharp \Lambda$, there exists a $\mathbb{Q}$-linear map $\psi_{n}: V_{n} \rightarrow \mathbb{R}$, where $V_{n}$ is the $\mathbb{Q}$-vector space generated by $\left\{\lambda_{1}, \lambda_{2}, \ldots, \lambda_{n}\right\}$, such that

$$
b_{j}=a_{j} e^{i \psi_{n}\left(\lambda_{j}\right)}, j=1, \ldots, n .
$$

In that case, we will write $f_{1} \stackrel{*}{\sim} f_{2}$.

The next important lemma allows us to prove that if a function $f_{2}$ is *equivalent (in the sense of Definition 7 ) to a function $f_{1}$ belonging to the space $G(\mathbb{R}, \mathbb{C})$, then $f_{2}$ also belongs to $G(\mathbb{R}, \mathbb{C})$.

Lemma 8 Let $f_{1}(t) \in G(\mathbb{R}, \mathbb{C})$ be an almost periodic function whose Fourier series is given by $\sum_{j \geq 1} a_{j} e^{i \lambda_{j} t}, a_{j} \in \mathbb{C}$, where $\left\{\lambda_{1}, \ldots, \lambda_{j}, \ldots\right\}$ is a set of distinct exponents. Consider $b_{j} \in \mathbb{C}$ such that $\sum_{j \geq 1} b_{j} e^{i \lambda_{j} t}$ and $\sum_{j \geq 1} a_{j} e^{i \lambda_{j} t}$ are ${ }^{*}$-equivalent. Then $\sum_{j \geq 1} b_{j} e^{i \lambda_{j} t}$ is the Fourier series associated with an almost periodic function $f_{2}(t) \in G(\mathbb{R}, \mathbb{C})$ so that $f_{1} \stackrel{*}{\sim} f_{2}$.

Proof. Take $\Lambda=\left\{\lambda_{1}, \ldots, \lambda_{j}, \ldots\right\}$. By hypothesis, $f_{1} \in \mathcal{F}_{G, \Lambda} \subset G(\mathbb{R}, \mathbb{C})$ is determined by a series of the form $\sum_{j \geq 1} a_{j} e^{i \lambda_{j} t}, a_{j} \in \mathbb{C}, \lambda_{j} \in \Lambda$. In virtue of [7, pp. 46-47] or [3, p. 105], let $P_{k}(t)=\sum_{j \geq 1} p_{j, k} a_{j} e^{i \lambda_{j} t}, k=1,2, \ldots$, be the Bochner-Fejér polynomials which converge to $f_{1}$ with respect to the topology of $G(\mathbb{R}, \mathbb{C})$ (in fact, $p_{j, k} \rightarrow 1$ as $k \rightarrow \infty$ ). Now take $\tau \in \mathbb{R}$, then $P_{k, \tau}(t)=\sum_{j \geq 1} p_{j, k} a_{j} e^{i \lambda_{j}(t+\tau)}, k=1,2, \ldots$, are the Bochner-Fejér polynomials which converge to $f_{1, \tau}(t):=f_{1}(t+\tau), t \in \mathbb{R}$. On the other hand, let $Q_{k}(t):=$ $\sum_{j \geq 1} p_{j, k} b_{j} e^{i \lambda_{j} t}$, with $t \in \mathbb{R}$ and $k=1,2, \ldots$ Given $\varepsilon>0$, by [15, Corollary 1], there exists a relatively dense set of real numbers $\tau$ such that

$$
\left|\sum_{j \geq 1} p_{j, k} a_{j} e^{i \lambda_{j}(t+\tau)}-\sum_{j \geq 1} p_{j, k} b_{j} e^{i \lambda_{j} t}\right|<\frac{\varepsilon}{3} \quad \forall t \in \mathbb{R} .
$$

Thus, by virtue of [3, p. 73], for this set of real numbers $\tau$ it is satisfied

$$
\begin{aligned}
& \| \sum_{j \geq 1} p_{j, k} a_{j} e^{i \lambda_{j}(t+\tau)}- \sum_{j \geq 1} p_{j, k} b_{j} e^{i \lambda_{j} t} \|_{G} \leq \\
& \sup \left\{\left|\sum_{j \geq 1} p_{j, k} a_{j} e^{i \lambda_{j}(t+\tau)}-\sum_{j \geq 1} p_{j, k} b_{j} e^{i \lambda_{j} t}\right|: t \in \mathbb{R}\right\} \leq \frac{\varepsilon}{3} .
\end{aligned}
$$


Furthermore, by the Bochner-Féjer property [3, p. 105], for every $\tau \in \mathbb{R}$ there exists $k_{0} \in \mathbb{N}$ such that

$$
\left\|P_{k, \tau}(t)-f_{1, \tau}(t)\right\|_{G}=\left\|\sum_{j \geq 1} p_{j, k} a_{j} e^{i \lambda_{j}(t+\tau)}-f_{1}(t+\tau)\right\|_{G} \leq \frac{\varepsilon}{3} \text { for each } k>k_{0} .
$$

Finally, by the property of $G$-normality applied to the sequence $\left\{f_{1, \tau}(t)\right\}_{\tau}$, where $\tau$ varies in the set above, we can extract a subsequence $\left\{f_{1, \tau_{m}}(t)\right\}_{m} \subset\left\{f_{1, \tau}(t)\right\}$ which converges to a certain function $f_{2}(t)$ with respect to the topology of $G(\mathbb{R}, \mathbb{C})$. This implies the existence of $m_{1} \in \mathbb{N}$ such that

$$
\left\|f_{2}(t)-f_{1}\left(t+\tau_{m}\right)\right\|_{G} \leq \frac{\varepsilon}{3} \text { for each } m>m_{1} .
$$

Now, take $\tau_{m}$ with $m>m_{1}$, and let $k>k_{0}$. We deduce from above that

$$
\begin{aligned}
\left\|\sum_{j \geq 1} p_{j, k} b_{j} e^{i \lambda_{j} t}-f_{2}(t)\right\|_{G} \leq \\
\leq\left\|\sum_{j \geq 1} p_{j, k} b_{j} e^{i \lambda_{j} t}-\sum_{j \geq 1} p_{j, k} a_{j} e^{i \lambda_{j}\left(t+\tau_{m}\right)}\right\|_{G}+ \\
+\| \sum_{j \geq 1} p_{j, k} a_{j} e^{i \lambda_{j}\left(t+\tau_{m}\right)}-f_{1}\left(t+\tau_{m}\right) \\
+\left\|f_{1}\left(t+\tau_{m}\right)-f_{2}(t)\right\|_{G} \leq \varepsilon,
\end{aligned}
$$

which implies that the sequence of trigonometric polynomials $\sum_{j \geq 1} p_{j, k} b_{j} e^{i \lambda_{j} t}$ converges to $f_{2}$ with respect to the topology of $G(\mathbb{R}, \mathbb{C})$ and, in fact, $f_{2} \in$ $G(\mathbb{R}, \mathbb{C})$ (recall that $G(\mathbb{R}, \mathbb{C})$ is defined as the closure of the trigonometric polynomials with respect to $\|\cdot\|_{G}$ ). Finally, by Definition 7 it is clear that $f_{1} \stackrel{*}{\sim} f_{2}$.

Note that the proof of the lemma above is notably different from that of [14, Lemma 2] and [15, Lemma 1]. Our next purpose is to prove that the equivalence classes of $\mathcal{F}_{G, \Lambda} /{ }^{*} \sim$ are closed, for which we will use the next remark and lemma.

Remark 9 If a sequence of trigonometric polynomials

$$
P_{k}(t)=\sum_{j \geq 1} a_{j}^{(k)} e^{i \lambda_{j} t}, k=1,2, \ldots,
$$

converge to a certain function $f(t)$ with respect to the topology of $G(\mathbb{R}, \mathbb{C})$, then the Fourier series of $f(t)$ is given by the formal limit of the series (5), i.e. the Forier series of $f(t)$ is given by $\sum_{j \geq 1} a_{j} e^{i \lambda_{j} t}$, where $a_{j}=\lim _{k \rightarrow \infty} a_{j}^{(k)}$ for each $j \geq 1$. Equivalently, $\left|M\left(P_{k}(t) e^{-i \lambda_{j} t}\right)-M\left(f(t) e^{-i \lambda_{j} t}\right)\right|$ tends to 0 as 
$k \rightarrow \infty$. Indeed, this result derives from [3, p. 21, Theorem] for the case $G(\mathbb{R}, \mathbb{C})=A P(\mathbb{R}, \mathbb{C})$, and the general case is deduced from [3, p. 105]. In fact, if $\left\{P_{k}(t)\right\}_{k}$ converges to $f(t)$ with respect to the topology of $G(\mathbb{R}, \mathbb{C})$, then it also converges with respect to the topology of $B(\mathbb{R}, \mathbb{C})$ and, by [3, p. 105], $\left|a_{j}^{(k)}-a_{j}\right|=\left|M\left(P_{k}(t) e^{-i \lambda_{j} t}\right)-M\left(f(t) e^{-i \lambda_{j} t}\right)\right|$ tends to 0 as $k \rightarrow \infty$.

Lemma 10 Given $\Lambda$ a set of exponents, let $\mathcal{G}$ be an equivalence class in $\mathcal{F}_{G, \Lambda} /{ }^{*}$. Let $\left\{f_{l}(t)\right\}_{l \geq 1} \subset \mathcal{G}$ and for each $l \geq 1$ consider $P_{l, k}(t), k=1,2, \ldots$, the Bochner-Fejér polynomials converging to $f_{l}(t)$ with respect to the topology of $G(\mathbb{R}, \mathbb{C})$. If $h(t) \in \mathcal{F}_{G, \Lambda}$ is the limit of a certain sequence $\left\{P_{l_{m}, k_{l_{m}}}(t)\right\}_{m \geq 1}$, with $\left\{l_{m}\right\}_{m \geq 1} \subset\{l\}_{l \geq 1}$ and $\left\{k_{l_{m}}\right\}_{m \geq 1} \subset\{k\}_{k \geq 1}$, then $h(t)$ belongs to $\mathcal{G}$.

Proof. Let $\left\{f_{l}\right\}_{l \geq 1}$ be a sequence in $\mathcal{G}$ and, for each $l=1,2, \ldots$, suppose that the Fourier series associated with $f_{l}(t)$ is given by

$$
\sum_{j \geq 1} a_{l, j} e^{i \lambda_{j} t} \text { with } a_{l, j} \in \mathbb{C} \backslash\{0\}, \lambda_{j} \in \Lambda .
$$

Also, given $l \geq 1$, let

$$
P_{l, k}(t)=\sum_{j \geq 1} p_{j, k} a_{l, j} e^{i \lambda_{j} t}, k=1,2, \ldots,
$$

be the Bochner-Fejér polynomials which converge to $f_{l}$ with respect to the topology of $G\left(\mathbb{R}, \mathbb{C}\right.$ ) (and which satisfy $p_{j, k} \rightarrow 1$ as $k \rightarrow \infty$ ) [3, p. 105, Theorem II]. Furthermore, let $\sum_{j \geq 1} b_{j} e^{i \lambda_{j} t}$ be the Fourier series of $h(t) \in \mathcal{F}_{G, \Lambda}$. By reductio ad absurdum, suppose that $h(t)$ is not in $\mathcal{G}$, which implies the existence of $n_{0} \in \mathbb{N}$, with $n_{0} \leq \sharp \Lambda$, satisfying

$$
b_{j}=a_{1, j} e^{i \psi_{n_{0}}\left(\lambda_{j}\right)}, j=1, \ldots, n_{0},
$$

is not satisfied for any $\mathbb{Q}$-linear map $\psi_{n_{0}}: V_{n_{0}} \rightarrow \mathbb{R}$ (see Definition 7). By hypothesis, the sequence $\left\{P_{l_{m}, k_{l_{m}}}(t)\right\}_{m \geq 1}$ converges to $h(t)$ with respect to the topology of $G(\mathbb{R}, \mathbb{C})$. From it, draw a subsequence $\left\{P_{l_{m, 1}, k_{l_{m, 1}}}(t)\right\}_{m \geq 1} \subset$ $\left\{P_{l_{m}, k_{l_{m}}}(t)\right\}_{m \geq 1}$ such that $p_{1, k_{l_{m, 1}}} \neq 0$ for each $m \geq 1$. Analogously, draw a subsequence $\left\{P_{l_{m, 2}, k_{l_{m, 2}}}(t)\right\}_{m \geq 1} \subset\left\{P_{l_{m, 1}, k_{l_{m, 1}}}(t)\right\}_{m \geq 1}$ such that $p_{2, k_{l_{m, 2}}} \neq 0$ (and $p_{1, k_{l_{m, 2}}} \neq 0$ ) for each $m \geq 1$. In this way, we can draw a subsequence $\left\{P_{l_{m, n_{0}}, k_{l_{m, n_{0}}}}(t)\right\}_{m \geq 1} \subset\left\{P_{l_{m}, k_{l_{m}}}(t)\right\}_{m \geq 1}$ such that $p_{j, k_{l_{m}, n_{0}}} \neq 0$ for each $j=$ $1,2, \ldots, n_{0}$ and $m \geq 1$ (this process is similar to that of Remark of [3, p. 51]). Now, since the polynomials

$P_{l_{m, n_{0}}, k_{l_{m, n_{0}}}}(t)=\sum_{j=1}^{n_{0}} p_{j, k_{l_{m, n_{0}}}} a_{l_{m, n_{0}}, j} e^{i \lambda_{j} t}+\sum_{j>n_{0}} p_{j, k_{l_{m, n_{0}}}} a_{l_{m, n_{0}}, j} e^{i \lambda_{j} t}, m=1,2, \ldots$

converge to $h(t)$, the Fourier series of $h(t)$ is the formal limit of the Fourier series of $P_{l_{m, n_{0}}, k_{l_{m, n_{0}}}}(t)$, i.e. $b_{j}=\lim _{m \rightarrow \infty} p_{j, k_{l_{m, n_{0}}}} a_{l_{m, n_{0}}, j}=\lim _{m \rightarrow \infty} a_{l_{m, n_{0}}, j}$ 
for each $j \geq 1$ (see Remark 9). This implies in particular that the functions

$$
Q_{l_{m, n_{0}}}(t):=\sum_{j=1}^{n_{0}} a_{l_{m, n_{0}}, j} e^{i \lambda_{j} t}, m=1,2, \ldots
$$

converge to $\sum_{j=1}^{n_{0}} b_{j} e^{i \lambda_{j} t}$. Furthermore, it is clear that $\left\{Q_{l_{m, n_{0}}}(t)\right\}_{m \geq 1}$ is a sequence of *-equivalent trigonometric polynomials and, by [15, Proposition 3], its limit is in the same equivalence class as $\sum_{j=1}^{n_{0}} a_{1, j} e^{i \lambda_{j} t}$, which represents a contradiction with (6).

With respect to the topology of $G(\mathbb{R}, \mathbb{C})$, we next prove that the equivalence classes of $\mathcal{F}_{G, \Lambda} / \stackrel{*}{\sim}$ are sequentially compact (and hence compact), which generalizes [15, Proposition 3]. The reader can observe that the proof given here is quite different from that of [15, Proposition 3].

Proposition 11 Let $\Lambda$ be a set of exponents and $f \in \mathcal{F}_{G, \Lambda}$. Then the equivalence class which contains $f$ is sequentially compact in $\mathcal{F}_{G, \Lambda} / \stackrel{*}{\sim}$.

Proof. Let $\mathcal{G}$ be the equivalence class which contains $f \in G(\mathbb{R}, \mathbb{C})$. We first note that, by Lemma $8, \mathcal{G}$ is included in $\mathcal{F}_{G, \Lambda} / \stackrel{*}{\sim}$. So, let $\left\{f_{l}\right\}_{l \geq 1}$ be a sequence in $\mathcal{G}$. Given $l \geq 1$, let $P_{l, k}(t), k=1,2, \ldots$, be the Bochner-Fejér polynomials which converge to $f_{l}$ with respect to the topology of $G(\mathbb{R}, \mathbb{C})[3$, p. 105 , Theorem II]. Given $\varepsilon>0$ and $l \geq 1$, let $k_{l} \geq 1$ such that

$$
\left\|P_{l, k}(t)-f_{l}(t)\right\|_{G}<\frac{\varepsilon}{4} \text { for each } k \geq k_{l} .
$$

Without loss of generality, suppose $k_{1} \leq \ldots \leq k_{l} \leq \ldots$, which particularly implies that

$$
\left\|P_{1, k_{l}}(t)-f_{1}(t)\right\|_{G}<\frac{\varepsilon}{4} \text { for each } l \geq 1 .
$$

Moreover, given $l \geq 1$ and $k_{l}$ as above, by Proposition 5 there exists $\tau_{l, k_{l}}$ such that

$$
\left\|P_{1, k_{l}}\left(t+\tau_{l, k_{l}}\right)-P_{l, k_{l}}(t)\right\|_{G}<\frac{\varepsilon}{4} .
$$

Now, consider the sequence $\left\{f_{1}\left(t+\tau_{l, k_{l}}\right)\right\}_{l \geq 1}$. Since the set of translates of $f_{1}$ is relatively compact (see the comments on the $G$-normality in the introduction), there exists a subsequence $\left\{f_{1}\left(t+\tau_{l(n), k_{l(n)}}\right)\right\}_{n \geq 1}$ which is convergent to a certain function $h(t) \in G(\mathbb{R}, \mathbb{C})$. Let $n_{0} \geq 1$ satisfy

$$
\left\|f_{1}\left(t+\tau_{l(n), k_{l(n)}}\right)-h(t)\right\|_{G}<\frac{\varepsilon}{4} \text { for each } n \geq n_{0} .
$$

So, for each $n \geq n_{0}$, we deduce from (7), (8), (9) and (10) that

$$
\begin{aligned}
& \left\|h(t)-f_{l(n)}(t)\right\|_{G} \leq \\
& \left\|h(t)-f_{1}\left(t+\tau_{l(n), k_{l(n)}}\right)\right\|_{G}+\left\|f_{1}\left(t+\tau_{l(n), k_{l(n)}}\right)-P_{1, k_{l(n)}}\left(t+\tau_{l(n), k_{l(n)}}\right)\right\|_{G}+ \\
& +\left\|P_{1, k_{l(n)}}\left(t+\tau_{l(n), k_{l(n)}}\right)-P_{l(n), k_{l(n)}}(t)\right\|_{G}+\left\|P_{l(n), k_{l(n)}}(t)-f_{l(n)}(t)\right\|_{G}<\varepsilon,
\end{aligned}
$$


which implies that $h(t)$ is an accumulation point of $\left\{f_{l}(t)\right\}_{l \geq 1}$. Furthermore, in virtue of

$$
\left\|h(t)-P_{l(n), k_{l(n)}}(t)\right\|_{G} \leq\left\|h(t)-f_{l(n)}(t)\right\|_{G}+\left\|f_{l(n)}(t)-P_{l(n), k_{l(n)}}(t)\right\|_{G},
$$

$h(t)$ is the limit of the Bochner-Féjer polynomials $\left\{P_{l(n), k_{l(n)}}(t)\right\}_{n \geq 1}$ and, by Lemma $10, h \in \mathcal{G}$.

Remark 12 We remind the reader that all these functions are regarded as members of a metric space and, hence, sequential compactness is the same as compactness (in the topology induced by the metric), and it implies being closed. Hence, as a consequence of Proposition 11, we can immediately generalize [15, Corollary 3].

Corollary 13 Let $\Lambda$ be a set of exponents and $f \in \mathcal{F}_{G, \Lambda}$. Then the limit points of the set of functions $\mathcal{T}_{f}=\left\{f_{\tau}(t):=f(t+\tau): \tau \in \mathbb{R}\right\}$ are functions which are *-equivalent to $f$.

Proof. Since it is plain that the functions included in the set given by $\mathcal{T}_{f}=$ $\left\{f_{\tau}(p):=f(t+\tau): \tau \in \mathbb{R}\right\}$ are in the same equivalence class as $f$ (see in [8, Section 4.2] the Fourier series of the translates of a function in $B(\mathbb{R}, \mathbb{C}))$, the result follows easily from Proposition 11.

Now Corollary 13 can be improved with the following result which proves that, given a function $f \in \mathcal{F}_{G, \Lambda}$, the limit points of the set of the translates $\mathcal{T}_{f}=\{f(t+\tau): \tau \in \mathbb{R}\}$ of $f$ are precisely the almost periodic functions which are ${ }^{*}$-equivalent to $f$.

Theorem 14 Let $\Lambda$ be a set of exponents, $\mathcal{G}$ an equivalence class in $\mathcal{F}_{G, \Lambda} /^{*}$ and $f \in \mathcal{G}$. Then the set of functions $\mathcal{T}_{f}=\left\{f_{\tau}(t):=f(t+\tau): \tau \in \mathbb{R}\right\}$ is dense in $\mathcal{G}$.

Proof. Let $f(t)$ be a function in the class $\mathcal{F}_{G, \Lambda}$. We know by Corollary 13 that the limit points of the set of functions $\mathcal{T}_{f}=\left\{f_{\tau}(t):=f(t+\tau): \tau \in \mathbb{R}\right\}$ are functions in $G(\mathbb{R}, \mathbb{C})$ which are *-equivalent to $f$. We next demonstrate that any function $h(t)$ which is ${ }^{*}$-equivalent to $f(t)$ is also a limit point of $\mathcal{T}_{f}$. Consider $\sharp \Lambda=\infty$ and let $\left\{P_{n}(t)\right\}_{n \geq 1}$ and $\left\{Q_{n}(t)\right\}_{n \geq 1}$ be the sequences of Bochner-Fejér polynomials which converge, with respect to the topology of $G(\mathbb{R}, \mathbb{C})$, to $f(t)$ and $h(t)$, respectively. Take $\varepsilon_{1}=\max \left\{\left\|f(t)-P_{1}(t)\right\|_{G},\left\|h(t)-Q_{1}(t)\right\|_{G}\right\}>$ 0 , then Proposition 5 assures the existence of a positive number $\tau_{1}$ satisfying $\left\|P_{1}\left(t+\tau_{1}\right)-Q_{1}(t)\right\|_{G} \leq \varepsilon_{1}$. Hence

$$
\begin{aligned}
& \left\|f\left(t+\tau_{1}\right)-h(t)\right\|_{G} \leq \\
& \qquad f\left(t+\tau_{1}\right)-P_{1}\left(t+\tau_{1}\right)\left\|_{G}+\right\| P_{1}\left(t+\tau_{1}\right)-Q_{1}(t) \|_{G}+ \\
& +\left\|Q_{1}(t)-h(t)\right\|_{G} \leq 3 \varepsilon_{1} .
\end{aligned}
$$


Similarly, take $\varepsilon_{2}=\max \left\{\left\|f(t)-P_{2}(t)\right\|_{G},\left\|h(t)-Q_{2}(t)\right\|_{G}\right\}>0$, then Proposition 5 assures the existence of $\tau_{2}>\tau_{1}$ such that $\left\|P_{2}\left(t+\tau_{2}\right)-Q_{2}(t)\right\|_{G} \leq \varepsilon_{2}$. Hence

$$
\begin{aligned}
& \left\|f\left(t+\tau_{2}\right)-h(t)\right\|_{G} \leq \\
& \qquad f\left(t+\tau_{2}\right)-P_{2}\left(t+\tau_{2}\right)\left\|_{G}+\right\| P_{2}\left(t+\tau_{2}\right)-Q_{2}(t) \|_{G} \\
& +\left\|Q_{2}(t)-h(t)\right\|_{G} \leq 3 \varepsilon_{2} .
\end{aligned}
$$

In general, by repeating this process, we can construct an increasing sequence $\left\{\tau_{n}\right\}_{n \geq 1}$ such that each $\tau_{n}$ satisfies that

$$
\left\|P_{n}\left(t+\tau_{n}\right)-Q_{n}(t)\right\|_{G} \leq \varepsilon_{n},
$$

with $\varepsilon_{n}=\max \left\{\left\|f(t)-P_{n}(t)\right\|_{G},\left\|h(t)-Q_{n}(t)\right\|_{G}\right\}$. Thus, from (11), it is accomplished that

$$
\begin{aligned}
\left\|f\left(t+\tau_{n}\right)-h(t)\right\|_{G} \leq & \left\|f\left(t+\tau_{n}\right)-P_{n}\left(t+\tau_{n}\right)\right\|_{G}+ \\
& +\left\|P_{n}\left(t+\tau_{n}\right)-Q_{n}(t)\right\|_{G}+\left\|Q_{n}(t)-h(t)\right\|_{G} \leq 3 \varepsilon_{n} .
\end{aligned}
$$

Finally, note that $\varepsilon_{n}$ tends to 0 when $n$ goes to $\infty$. Consequently, the sequence of functions $\left\{f\left(t+\tau_{n}\right)\right\}_{n \geq 1}$ converges to $h(t)$ with respect to the topology of $G(\mathbb{R}, \mathbb{C})$ and the result holds.

Corollary 15 Let $f \in G(\mathbb{R}, \mathbb{C})$ and $h \stackrel{*}{\sim} f$. There exists an increasing unbounded sequence $\left\{\tau_{n}\right\}_{n \geq 1}$ of positive numbers such that the sequence of functions $\left\{f\left(t+\tau_{n}\right)\right\}_{n \geq 1}$ converges with respect to the topology of $G(\mathbb{R}, \mathbb{C})$ to $h(t)$. In fact, given $\varepsilon>0$, it is accomplished that

i) if $G=A P, G=S^{p}$ or $G=W^{p}$, there exists a relatively dense set of positive numbers $\{\tau\}$ such that

$$
\|f(t+\tau)-h(t)\|_{G} \leq \varepsilon \forall \tau \in\{\tau\}
$$

ii) if $G=B^{p}$ there exists a satisfactorily uniform set of real numbers $\{\tau\}$ such that

$$
\|f(t+\tau)-h(t)\|_{B^{p}} \leq \varepsilon \forall \tau \in\{\tau\} .
$$

Proof. Let $f \in G(\mathbb{R}, \mathbb{C})$, then $f \in \mathcal{F}_{G, \Lambda}$ for some set $\Lambda$ of exponents. First of all, fixed $a \in \mathbb{R}$, let $f_{a}(t)$ denote the function $f_{a}(t):=f(t+a), t \in \mathbb{R}$. Consider $\mathcal{G}$ an equivalence class in $\mathcal{F}_{G, \Lambda} / \stackrel{*}{\sim}$ so that $f \in \mathcal{G}$ and let $h \stackrel{*}{\sim} f$. Thus, by Theorem 14 (see also its proof), there exists an increasing unbounded sequence $\left\{\delta_{n}\right\}_{n \geq 1}$ of positive numbers such that the sequence of functions $\left\{f_{\delta_{n}}(t)\right\}_{n \geq 1}$ converges with respect to the topology of $G(\mathbb{R}, \mathbb{C})$ to $h(t)$. Equivalently, given $\varepsilon>0$ there exists $n_{0} \in \mathbb{N}$ such that

$$
\left\|f_{\delta_{n}}(t)-h(t)\right\|_{G} \leq \varepsilon / 2 \forall n \geq n_{0} .
$$

To prove the second statement, we will use the fact that $f$ is a $G$-a.p. function: 
a) If $G=A P$ or $G=S^{p}$, there exists a relatively dense set $\left\{\tau_{k}\right\} \subset \mathbb{R}$ such that

$$
\left\|f_{\tau_{k}}(t)-f(t)\right\|_{G} \leq \varepsilon / 2 \forall \tau_{k} \in\left\{\tau_{k}\right\} .
$$

Therefore, from (12) and (13), each $\tau_{k}$ satisfies

$$
\begin{aligned}
\left\|f_{\delta_{n}+\tau_{k}}(t)-h(t)\right\|_{G} \leq\left\|f_{\delta_{n}+\tau_{k}}(t)-f_{\delta_{n}}(t)\right\|_{G}+\left\|f_{\delta_{n}}(t)-h(t)\right\|_{G}= \\
\left\|f_{\tau_{k}}\left(t+\delta_{n}\right)-f\left(t+\delta_{n}\right)\right\|_{G}+\left\|f_{\delta_{n}}(t)-h(t)\right\|_{G} \leq \varepsilon \quad \forall n \geq n_{0} .
\end{aligned}
$$

b) If $G=W^{p}$, there exists a relatively dense set $\left\{\tau_{k}\right\} \subset \mathbb{R}$ and $L_{0}>0$ such that

$$
\left\|f_{\tau_{k}}(t)-f(t)\right\|_{S_{l}^{p}} \leq \varepsilon / 2 \forall \tau_{k} \in\left\{\tau_{k}\right\} \forall l \geq L_{0} .
$$

Therefore, from (12), (14) and [3, p. 73] (or [2, p. 170]), each $\tau_{k}$ and $l \geq L_{0}$ satisfies

$$
\begin{aligned}
\left\|f_{\delta_{n}+\tau_{k}}(t)-h(t)\right\|_{W^{p}} \leq\left\|f_{\delta_{n}+\tau_{k}}(t)-f_{\delta_{n}}(t)\right\|_{W^{p}}+\left\|f_{\delta_{n}}(t)-h(t)\right\|_{W^{p}}= \\
\left\|f_{\tau_{k}}\left(t+\delta_{n}\right)-f\left(t+\delta_{n}\right)\right\|_{W^{p}}+\left\|f_{\delta_{n}}(t)-h(t)\right\|_{W^{p}} \leq \\
\left\|f_{\tau_{k}}\left(t+\delta_{n}\right)-f\left(t+\delta_{n}\right)\right\|_{S_{l}^{p}}+\frac{\varepsilon}{2} \leq \frac{\varepsilon}{2}+\frac{\varepsilon}{2}=\varepsilon \quad \forall n \geq n_{0} .
\end{aligned}
$$

Hence we have proved i).

c) If $G=B^{p}$, there exists a satisfactorily uniform set $\left\{\tau_{k}\right\} \subset \mathbb{R}$ such that

$$
\left\|f_{\tau_{k}}(t)-f(t)\right\|_{B^{p}} \leq \varepsilon / 2 \forall \tau_{k} \in\left\{\tau_{k}\right\} .
$$

Therefore, from (12) and (15), each $\tau_{k}$ satisfies

$$
\begin{array}{r}
\left\|f_{\delta_{n}+\tau_{k}}(t)-h(t)\right\|_{B^{p}} \leq\left\|f_{\delta_{n}+\tau_{k}}(t)-f_{\delta_{n}}(t)\right\|_{B^{p}}+\left\|f_{\delta_{n}}(t)-h(t)\right\|_{B^{p}}= \\
\left\|f_{\tau_{k}}\left(t+\delta_{n}\right)-f\left(t+\delta_{n}\right)\right\|_{B^{p}}+\left\|f_{\delta_{n}}(t)-h(t)\right\|_{B^{p}} \leq \varepsilon \quad \forall n \geq n_{0},
\end{array}
$$

which proves ii).

We have seen in the introduction that the $G$-a.p. functions satisfy the Bochner-type property consisting of the relative compactness of the set $\{f(t+$ $\tau)\}, \tau \in \mathbb{R}$, associated with an arbitrary function $f \in G(\mathbb{R}, \mathbb{C})$. As an important consequence of Theorem 14, we next refine this property in the sense that we show that the condition of $G$-almost periodicity of a function $f(t) \in G(\mathbb{R}, \mathbb{C})$ implies that every sequence $\left\{f\left(t+\tau_{n}\right)\right\}, \tau_{n} \in \mathbb{R}$, of translates of $f$ has a subsequence that converges with the topology of $G(\mathbb{R}, \mathbb{C})$ to a function which is *-equivalent to $f$.

Corollary 16 If $f \in G(\mathbb{R}, \mathbb{C})$, then the closure of its set of translates coincides with its equivalence class and it is compact.

Proof. It is straightforward from Theorem 14 and Proposition 11. 
Remark 17 The main results of this paper, Theorem 14 and Corollary 16, have been formulated for the cases of $G$-a.p. functions, where $G$ could be $A P, S^{p}$, $W^{p}$ or $B^{p}$, with $1 \leq p<\infty$. However, we can go further by generalizing these results to every existing space $T(\mathbb{R}, \mathbb{C})$ of functions $f: \mathbb{R} \rightarrow \mathbb{C}$, which can be associated with a Fourier series, satisfying the appropriate conditions used in this work. In particular, we have the following conditions:

i) The space $T(\mathbb{R}, \mathbb{C})$ is defined as the closure of the trigonometric polynomials with respect to a certain topology $\|\cdot\|_{T}$;

ii) It is satisfied the Bochner-type property consisting of the relative compactness of the set $\{f(t+\tau)\}, \tau \in \mathbb{R}$, associated with an arbitrary function $f \in T(\mathbb{R}, \mathbb{C}) ;$

iii) $T(\mathbb{R}, \mathbb{C})$ contains $A P_{1}(\mathbb{R}, \mathbb{C})$ (and hence it is satisfied that $\left\|f_{1}(t)-f_{2}(t)\right\|_{T} \leq$ $\left.\left\|f_{1}(t)-f_{2}(t)\right\|_{A P_{1}}\right)$, where $A P_{1}(\mathbb{R}, \mathbb{C})$ consists of all functions $f$ from $\mathbb{R}$ into $\mathbb{C}$ representable in the form $\sum_{j=1}^{\infty} a_{j} e^{i \lambda_{j} t}, \lambda_{j} \in \mathbb{R}, a_{j} \in \mathbb{C}$, with $\|f\|_{A P_{1}}:=\sum_{j=1}^{\infty}\left|a_{j}\right|<\infty$ (therefore $\left.A P_{1}(\mathbb{R}, \mathbb{C}) \subset A P(\mathbb{R}, \mathbb{C})\right)$.

iv) There exists a method of summation in the style of that of Bochner-Féjer. That is, every $f \in T(\mathbb{R}, \mathbb{C})$, associated with a Fourier series $\sum_{k \geq 1} a_{k} e^{i \lambda_{k} t}$, can be approximated by polynomials of the form $P_{k}^{f}(t)=\sum_{j \geq 1} p_{j, k} a_{j} e^{i \lambda_{j} t}$, where the terms $p_{j, k}$ only depend on the frequency $\lambda_{j}$ (not on the function f).

Acknowledgements. The first author's research was supported by PGC2018097960-B-C22 (MCIU/AEI/ERDF, UE)

\section{References}

[1] T.M. Apostol, Modular functions and Dirichlet series in number theory (Springer-Verlag, New York, 1990).

[2] J. Andres, A.M. Bersani and A.M. Grande, Hierarchy of almost-periodic function spaces, Rendiconti di Matematica, Serie VII 26 (2006) 121-188.

[3] A.S. Besicovitch, Almost periodic functions (Dover, New York, 1954).

[4] S. Bochner, A new approach to almost periodicity, Proc. Nat. Acad. Sci. 48 (1962) 2039-2043.

[5] H. Bohr, Almost periodic functions, (Chelsea, New York, 1951).

[6] H. Bohr, Contribution to the theory of almost periodic functions, Det Kgl. danske Videnskabernes Selskab. Matematisk-fisiske meddelelser Bd. XX. Nr. 18, Copenhague, 1943.

[7] H. Bohr and E. Fœlner, On some types of functional spaces, Acta Math. 76 (1945) 31-155. 
[8] C. Corduneanu, Almost periodic oscillations and waves, (Springer, New York, 2009).

[9] E. Fœlner, On the structure of generalized almost periodic function, Danske Vid. Selsk. Math. Phys., Medd. 21 (11) (1945) 1-30.

[10] W. Huang and Y. Yi, Almost periodically forced circle flows, J. Funct. Anal. 257 (3) (2009) 832-902.

[11] B. Jessen, Some aspects of the theory of almost periodic functions, in Proc. Internat. Congress Mathematicians Amsterdam, 1954, Vol. 1, North-Holland, 1954, pp. 304-351.

[12] L. Qi and R. Yuan, A generalization of Bochner's theorem and its applications in the study of impulsive differential equations, J. Dynam. Differential Equations, 31 (4) (2019) 1955-1985.

[13] R. Ortega and M. Tarallo, Almost periodic linear differential equations with non-separated solutions, J. Funct. Anal. 237 (2) (2006) 402-426.

[14] J.M. Sepulcre and T. Vidal, Almost periodic functions in terms of Bohr's equivalence relation, Ramanujan J. 46 (1) (2018) 245-267. Corrigendum, ibid, 48 (3), 685-690 (2019).

[15] J.M. Sepulcre and T. Vidal, Bohr's equivalence relation in the space of Besicovitch almost periodic functions, Ramanujan J. 49 (3) (2019) 625-639.

[16] W. Stepanov, Über einige Verallgemeinerungen der fastperiodischen Funktionen, Math. Ann. 95 (1926) 473-498. 